\title{
Thermoelectric and Interference Effects in a Kondo-Correlated Quantum Dot with Rashba Spin-Orbit Coupling
}

\author{
Ł. KARWACKI ${ }^{a, *}$, P. TROCHA ${ }^{a}$ AND J. BARNAS ${ }^{a, b}$ \\ ${ }^{a}$ Faculty of Physics, Adam Mickiewicz University, Umultowska 85, 61-614 Poznań, Poland \\ ${ }^{b}$ Institute of Molecular Physics, Polish Academy of Sciences, M. Smoluchowskiego 17, 60-179 Poznań, Poland
}

\begin{abstract}
Thermoelectric effects in spin-polarized transport through a strongly correlated quantum dot coupled to two ferromagnetic leads is investigated theoretically by means of the finite- $U$ slave boson technique. The dot is coupled to the leads both symmetrically via the spin-conserving tunneling processes and asymmetrically via spin-nonconserving Rashba-induced tunneling terms. The asymmetric coupling leads to various interference effects which modify transport properties. We have analyzed such thermoelectric coefficients like the electronic contribution to heat conductance $\kappa$, thermopower $S$, and thermoelectric efficiency $Z T$.
\end{abstract}

DOI: $10.12693 /$ APhysPolA.124.901

PACS: 73.23.-b, 73.63.Kv 72.15.Qm, 85.35.Ds

\section{Introduction}

Thermoelectric phenomena in nanostructures are of current interest due to the possibility of enhanced thermoelectric efficiency. Moreover, the interplay of spin, charge and heat transport leads to novel spin related thermoelectric effects, like for instance the spin Seebeck effect. Among various nanostructures, quantum dots are ones of the smallest artificial nanoelectronics elements, which provide interesting playground for single-electron thermoelectrics, and where the effects due to electron correlations, quantum confinement, and quantum interference can significantly modify transport properties of the system.

Most of the theoretical works on transport properties of quantum dots concerned the limit of spin-conserving tunneling processes. However, introducing the Rashba spin-orbit coupling to the system leads to spin precession and spin-flip processes. These processes generally lift the spin degeneracy [1] usually occurring in the absence of magnetic field (external or internal) and spin-orbit interactions. Both spin precession and spin-flip processes induced by the Rashba spin-orbit coupling have been studied recently in the context of possible applications in spintronics devices [2, 3]. It has been shown, among others, that strong Rashba coupling leads to intersubband mixing of electron waves corresponding to different spin orientations, which dominates over the spin precession [4-6] and results in the interference effects with characteristic Fano and Dicke lines [7].

In this paper we investigate the system with the Rashba spin-orbit coupling in the Kondo regime. The interplay of the Kondo correlations and spin, charge and heat currents is known to lead to interesting thermoelec-

*corresponding author; e-mail: karwacki@amu.edu.pl tric phenomena [8-10]. In the case considered, the dot is coupled symmetrically to the leads via a spin-conserving term and asymmetrically via spin-nonconserving Rashba-induced tunneling. To calculate the transport characteristics we apply the slave boson technique in the mean field approximation.

\section{Theoretical description}

The system investigated in this paper consists of a quantum dot with single orbital energy level, $\varepsilon_{\sigma}=$ $\varepsilon_{d}-\hat{\sigma} B$, where $B$ is the magnetic field measured in the energy units. The dot is connected to two ferromagnetic leads via spin-conserving couplings, described by the tunneling amplitudes $V_{\boldsymbol{k} \beta \sigma}$, (where $\beta=\mathrm{L}$, R for the left and right lead, respectively), and spin-nonconserving tunneling due to the Rashba spin-orbit interaction, described by $V_{\boldsymbol{k} \beta \sigma}^{\text {so }}$. We consider the limit of strong Rashba coupling $[2,4,6]$, when spin-flip effects dominate. The system can be described with the Hamiltonian

$$
\begin{aligned}
H & =\sum_{\boldsymbol{k} \beta \sigma} \varepsilon_{\boldsymbol{k} \beta \sigma} c_{\boldsymbol{k} \beta \sigma}^{\dagger} c_{\boldsymbol{k} \beta \sigma}+\sum_{\sigma} \varepsilon_{\sigma} d_{\sigma}^{\dagger} d_{\sigma}+U n_{\uparrow} n_{\downarrow} \\
& +\sum_{\boldsymbol{k} \beta \sigma}\left[V_{\boldsymbol{k} \beta \sigma} c_{\boldsymbol{k} \beta \sigma}^{\dagger} d_{\sigma}-V_{\boldsymbol{k} \beta \bar{\sigma}}^{\mathrm{so}} c_{\boldsymbol{k} \beta \bar{\sigma}}^{\dagger}\left(\mathrm{i} \boldsymbol{\sigma}_{x}\right)_{\sigma \bar{\sigma}} d_{\sigma}+\text { H.c. }\right],
\end{aligned}
$$

where the first term describes the leads, the next two terms correspond to the dot (with $U$ being the Coulomb energy), while the last term describes couplings to the leads. In the following we assume $V_{\boldsymbol{k} \beta \sigma}$ to be independent of $\boldsymbol{k}$ and introduce the coupling parameter $\Gamma_{\beta \sigma}$ defined as $\Gamma_{\beta \sigma}=2 \pi\left|V_{\beta \sigma}\right|^{2} \rho_{\beta \sigma}$, where $\rho_{\beta \sigma}$ is the spin dependent density of states in the lead $\beta$. Similarly, we define $\Gamma_{\beta \sigma}^{\text {so }}=$ $2 \pi\left|V_{\beta \sigma}^{\text {so }}\right|^{2} \rho_{\beta \sigma}$. Furthermore, we write $\Gamma_{\beta \sigma}=\left(1 \pm \sigma p_{\beta}\right) \Gamma_{\beta}$, where $p_{\beta}$ is the spin polarization of the lead $\beta$ and $\Gamma_{\beta}$ is the coupling parameter. Following this, we introduce $\Gamma_{\beta \sigma}^{\mathrm{so}}=\left(1 \pm \sigma p_{\beta}\right) \Gamma_{\beta}^{\mathrm{so}}$, and write $\Gamma_{\beta}^{\mathrm{so}}=q_{\beta} \Gamma_{\beta}$, where $q_{\beta}$ are the parameters which describe relative strength of the Rashba interaction.

To describe the Kondo correlations we use the finite- $U$ slave-boson method $[11,12]$. This method provides a reliable description of electron transport through quantum 
dot systems in the low temperature regime, $T \ll T_{\mathrm{K}}$, where $T_{\mathrm{K}}$ is the so-called Kondo temperature. The method relies on the replacement of bare dot operators $d_{\sigma}$ (and also their conjugate counterparts) by the product of pseudofermion and projection operators, $f_{\sigma} z_{\sigma}$, where $z_{\sigma}=e p_{\sigma}^{\dagger}+p_{\bar{\sigma}}^{\dagger} d$. The operators $e, p_{\sigma}$, and $d$ are projection operators on empty, singly occupied with spin $\sigma$, and doubly occupied states, respectively. These operators obey the bosonic commutation relations. Furthermore, in the mean field approximation these operators are replaced by their mean values. Such a transformation leads to a Hamiltonian with modified dot level energy $\tilde{\varepsilon}_{\sigma}=\varepsilon_{\sigma}+\lambda_{\sigma}^{(2)}$, modified tunneling amplitudes $\tilde{V}_{\boldsymbol{k} \beta \sigma}^{(\mathrm{so})}=V_{\boldsymbol{k} \beta \sigma}^{(\mathrm{so})} z_{\sigma}$ (and also $\left.\tilde{V}_{\boldsymbol{k} \beta \sigma}=V_{\boldsymbol{k} \beta \sigma} z_{\sigma}\right)$, and also introduces an additional constant term $E_{d}=U d^{2}+$ $\lambda^{(1)}\left(e^{2}+p_{\uparrow}^{2}+p_{\downarrow}^{2}+d^{2}\right)-\sum_{\sigma} \lambda_{\sigma}^{(2)}\left(p_{\sigma}^{2}+d^{2}\right)$ responsible for conservation of the state space of the dot, in which $\lambda^{(1)}$ and $\lambda_{\sigma}^{(2)}$ are certain parameters. These parameters, as well as the mean values $e, p_{\sigma}$ and $d$, have to be determined numerically via a set of self-consistent equations

$$
\begin{aligned}
& \sum_{\sigma} \partial_{e} \ln z_{\sigma} K_{\sigma}+2 \lambda^{(1)} e=0, \\
& \sum_{\sigma} \partial_{p_{\sigma^{\prime}}} \ln z_{\sigma} K_{\sigma}+2\left(\lambda^{(1)}-\lambda_{\sigma^{\prime}}^{(2)}\right) p_{\sigma^{\prime}}=0, \\
& \sum_{\sigma} \partial_{d} \ln z_{\sigma} K_{\sigma}+2\left(U+\lambda^{(1)}-\sum_{\sigma} \lambda_{\sigma}^{(2)}\right) d=0,
\end{aligned}
$$

where $K_{\sigma}=(1 / \pi) \int \mathrm{d} \varepsilon\left(\varepsilon-\tilde{\varepsilon}_{\sigma}\right) G_{\sigma}^{<}$, with $G_{\sigma}^{<}$denoting the lesser Green function. To find $G_{\sigma}^{<}$we need to know first the retarded (advanced) Green functions $G_{\sigma}^{\mathrm{r}(\mathrm{a})}$. These can be derived from the Dyson equation $\boldsymbol{G}^{\mathrm{r}}=\left(\boldsymbol{g}_{0}^{\mathrm{r}}-\boldsymbol{\Sigma}^{\mathrm{r}}\right)^{-1}$, where $g_{0 \sigma \sigma^{\prime}}^{\mathrm{r}}=\delta_{\sigma \sigma^{\prime}}\left(\varepsilon-\tilde{\varepsilon}_{\sigma}+\mathrm{i} 0^{+}\right)^{-1}$ and $\Sigma^{\mathrm{r}}=\Sigma_{\mathrm{L}}^{\mathrm{r}}+\boldsymbol{\Sigma}_{\mathrm{R}}^{\mathrm{r}}$ is the self-energy matrix, with the $\beta$ term $(\beta=\mathrm{L}, \mathrm{R})$ given by

$$
\begin{aligned}
& \Sigma_{\beta, 11}^{\mathrm{r}}=(\mathrm{i} / 2)\left[\tilde{\Gamma}_{\beta \uparrow}+\tilde{\Gamma}_{\beta \downarrow}^{\mathrm{so}}\right], \\
& \Sigma_{\beta, 22}^{\mathrm{r}}=(i / 2)\left[\tilde{\Gamma}_{\beta \downarrow}+\tilde{\Gamma}_{\beta \uparrow}^{\mathrm{so}}\right] \\
& \Sigma_{\beta, 12}^{\mathrm{r}}=-\Sigma_{\beta, 21}^{\mathrm{r}}=(1 / 2)\left[\sqrt{\tilde{\Gamma}_{\beta \uparrow} \tilde{\Gamma}_{\beta \uparrow}^{\mathrm{so}}}-\sqrt{\widetilde{\Gamma}_{\beta \downarrow} \widetilde{\Gamma}_{\beta \downarrow}^{\mathrm{so}}}\right]
\end{aligned}
$$

Here, $\tilde{\Gamma}_{\beta \sigma}$ and $\tilde{\Gamma}_{\beta \sigma}^{\mathrm{so}}$ are the renormalized coupling parameters. The lesser (Keldysh) Green function can be then calculated from the formula $\boldsymbol{G}^{<}=\mathrm{i}\left(f_{\mathrm{L}} \boldsymbol{G}^{\mathrm{r}} \tilde{\boldsymbol{\Gamma}}_{\mathrm{L}} \boldsymbol{G}^{\mathrm{a}}+\right.$ $\left.f_{\mathrm{R}} \boldsymbol{G}^{\mathrm{r}} \tilde{\boldsymbol{\Gamma}}_{\mathrm{R}} \boldsymbol{G}^{\mathrm{a}}\right)$, where $\tilde{\boldsymbol{\Gamma}}_{\beta}$ is related to the self-energy $\boldsymbol{\Sigma}_{\beta}$ as $\tilde{\boldsymbol{\Gamma}}_{\beta}=-\mathrm{i} \boldsymbol{\Sigma}_{\beta}$, while $f_{\mathrm{L}(\mathrm{R})}$ are the Fermi-Dirac distributions in respective leads.

To describe transport properties of the system, we calculate first the transmission function, $T(\varepsilon)=$ $\operatorname{Tr}\left(\boldsymbol{G}^{\mathrm{a}} \tilde{\boldsymbol{\Gamma}}_{\mathrm{R}} \boldsymbol{G}^{\mathrm{r}} \tilde{\boldsymbol{\Gamma}}_{\mathrm{L}}\right)$. It is then directly related to the basic transport coefficients via the appropriate transport integrals $L_{n(=0,1,2)}=-(1 / h) \int \mathrm{d} \varepsilon(\varepsilon-\mu)^{n}\left(\partial_{\varepsilon} f\right) T(\varepsilon)$. The coefficients calculated are: electrical conductance $G=$ $e^{2} L_{0}$, electronic contribution to the heat conductance $\kappa=(1 / T)\left(L_{2}-L_{1}^{2} / L_{0}\right)$, the Seebeck coefficient (thermopower $S=-(1 /|e| T)\left(L_{1} / L_{0}\right)$, and the dimensionless figure of merit $Z T=G S^{2} T / \kappa$.

\section{Numerical results and discussion}

The numerical results presented below are for symmetric spin-conserving coupling of the dot to the leads, $\Gamma_{\mathrm{L}}=\Gamma_{\mathrm{R}}=\Gamma$. Apart from this, in calculations we assumed $k_{\mathrm{B}} T=0.009 \Gamma$ and $U=6 \Gamma$. The linear electrical conductance and the linear thermoelectric coefficients have been calculated assuming absence of spin accumulation in the leads.

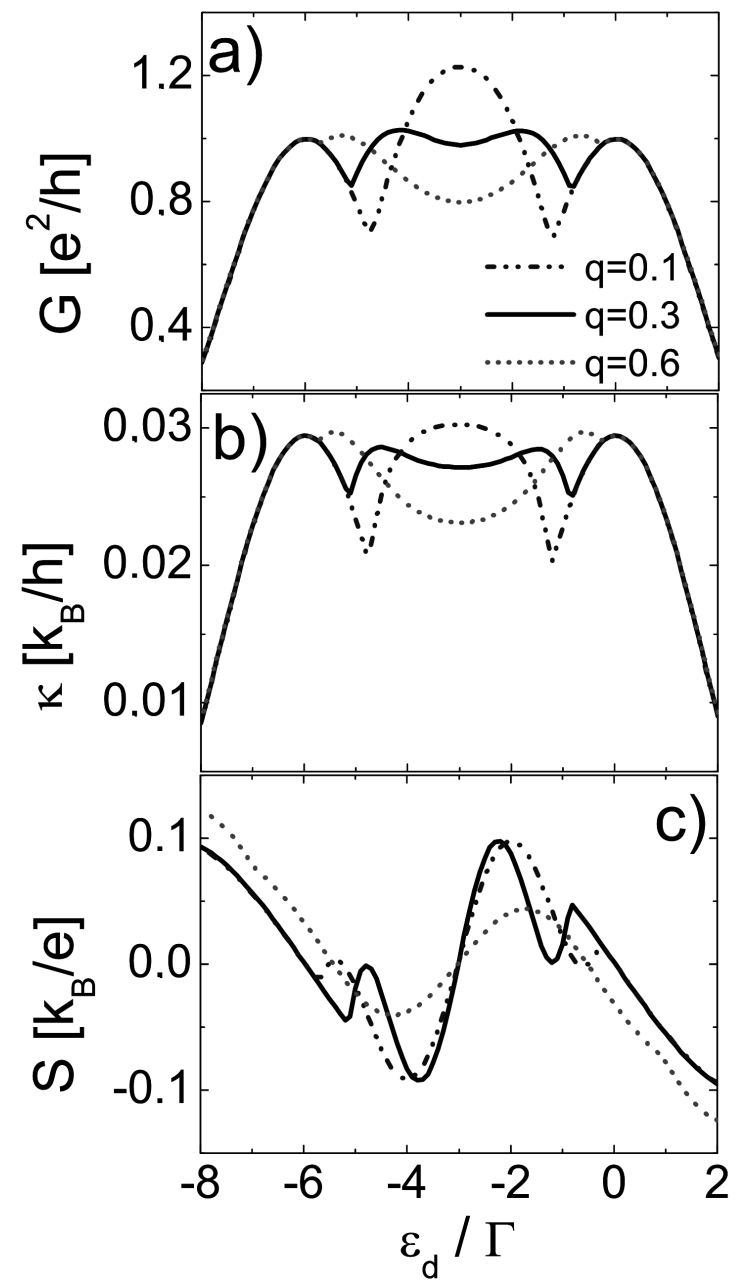

Fig. 1. Electrical conductance $G$ (a), electronic contribution to the heat conductance $\kappa(\mathrm{b})$, and thermopower $S$ (c), calculated as a function of the dot's level energy for indicated values of the parameter $q$. The other parameters: $p=0.9$, and $B=0$.

The calculated transport and thermoelectric coefficients are shown in Fig. 1 as a function of the dot's level energy and for different values of the parameter $q_{\mathrm{R}}=q$ and zero external magnetic field, $B=0$. Spin polarization of both leads was assumed the same and relatively large, $p_{\mathrm{L}}=p_{\mathrm{R}}=p=0.9$. Furthermore, no Rashba spin-orbit coupling between the dot and the left electrode was assumed, $q_{\mathrm{L}}=0$. The electrical conductance $G$ is shown in Fig. 1a. The dependence on the level energy has the shape that reveals two Hubbard resonance 
peaks and the Kondo peak centered at the particle-hole symmetric point, $\varepsilon_{d}=-U / 2$. When $q$ increases, the spin degeneracy of the dot's level is lifted, which results in splitting of the central peak. Finally, when $q$ is sufficiently large, this splitting leads to suppression of the Kondo effect.

Electronic contribution to the heat conductance, $\kappa$, follows qualitatively the behavior of the electrical conductance. This is a general tendency at low temperatures. In turn, the Seebeck coefficient $S$ (or thermopower) shown in Fig. 1c varies with the position of the dot's level in a more complex way, changing sign several times. Generally, the thermopower at low temperatures changes sign at the points where the transmission as a function of the dot's level reaches either minimum or maximum. Around such points, the current due to electrons is usually compensated by that due to holes and no voltage is generated by a temperature gradient. The transmission behavior is reflected in the conductance shown in Fig. 1a. Indeed, one can see that the thermopower shown in Fig. 1c vanishes at the points (or very close), where the conductance reaches extremum points. As a rule, the Seebeck coefficient vanishes in the particle-hole symmetric point, $\varepsilon_{d}=-U / 2$.

Figure 2 presents transport coefficients as a function of dot's level energy for different values of the magnetic field $B$, in the case of full leads' spin polarization, $p=1$, $q_{\mathrm{L}}=0$, and $q=q_{\mathrm{R}}=0.6$. When the leads are fully polarized, the Kondo effect is completely suppressed for $q=0$, as follows from the electrical and heat conductances shown in Fig. $2 \mathrm{a}$ and b, respectively. Introduction of the Rashba interaction results in a small splitting of the Hubbard peaks. Moreover, magnetic field leads to some asymmetry in the transmission coefficient and transport properties of the system.

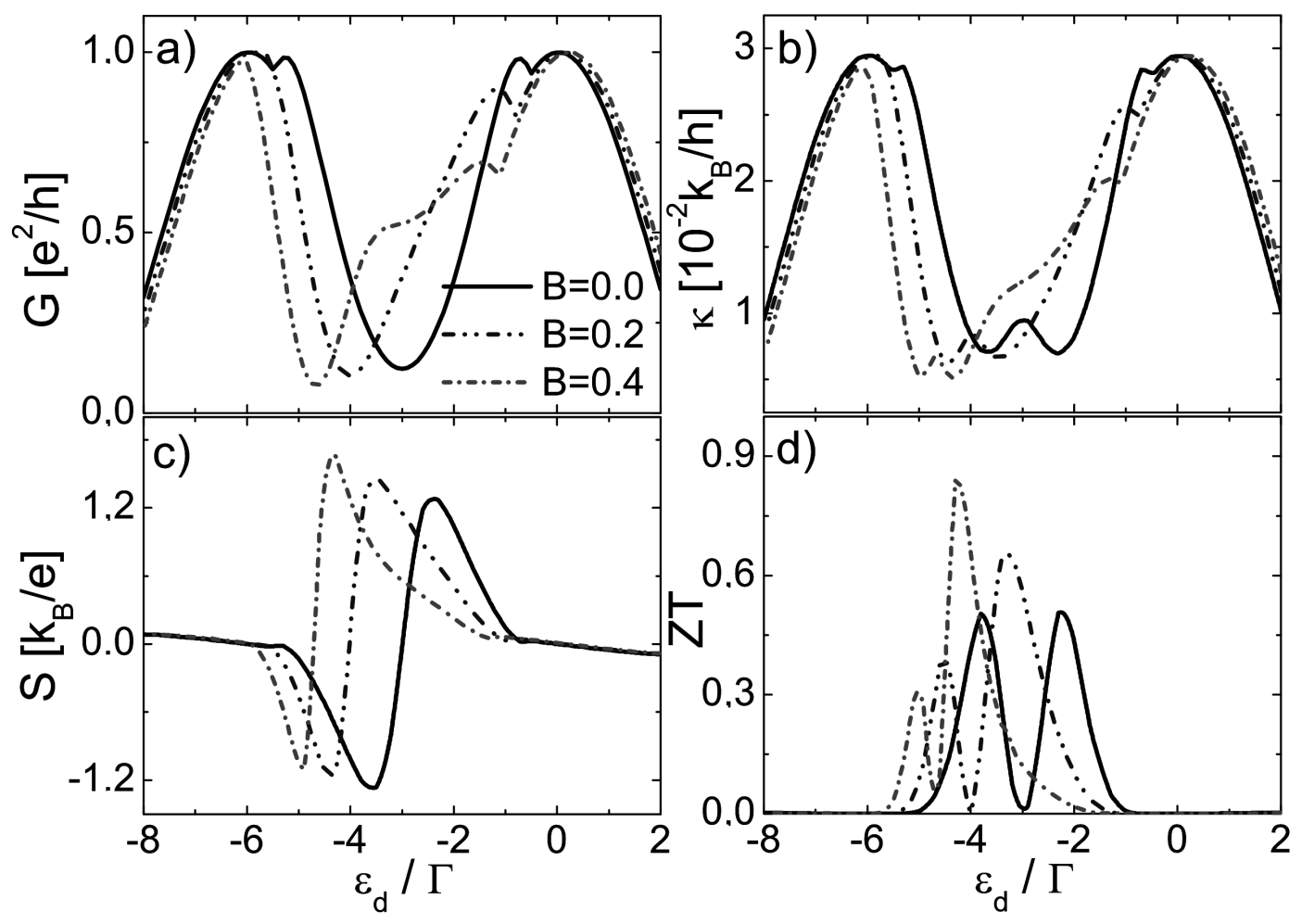

Fig. 2. Electrical conductance $G$ (a), electronic contribution to heat conductance $\kappa$ (b), thermopower $S$ (c), and figure of merit $Z T$ (d), calculated as a function of the dot's level energy for indicated values of the magnetic field $B$. The other parameters: $p=1$, and $q=0.6$.

Figure 2c shows that the curve presenting the Seebeck coefficient becomes shifted by a magnetic field towards lower energy level energy. This asymmetry results from the interplay of the Rashba-induced effective field and the external magnetic field. Similar shift and asymmetry appears also in the figure of merit presented in Fig. 2d. It is worth to note that a relatively strong magnetic field, $B / \Gamma=0.4$, leads to high asymmetry and consequently to some enhancement of the figure of merit to the values near the border of applicability, $Z T=1$.

\section{Conclusions}

We have analyzed thermoelectric transport in the linear response regime through a strongly correlated quantum dot connected to ferromagnetic electrodes via spin-conserving and spin-nonconserving couplings. Two situations have been analyzed: (i) high polarization of the 
leads, $p=0.9$, without external magnetic field, and (ii) full spin polarization, $p=1$, with magnetic field present. In the former case the lifting of the spin degeneracy due to the Rashba interaction results in suppression of the Kondo effect and in a weak enhancement of the thermopower and figure of merit. In turn, in the former case we have shown that the presence of magnetic field in fully polarized case results in a significant enhancement of the figure of merit due to the asymmetry induced by the interplay of Rashba-induced effective field, external magnetic field, and the electron correlations.

\section{Acknowledgments}

This work was supported by the National Science Center in Poland as the project No. DEC-2012/04/A/ST3/ 00372 .

\section{References}

[1] Y.A. Bychkov, E.I. Rashba, J. Phys. C 17, 6039 (1984).
[2] S. Datta, B. Das, Appl. Phys. Lett. 56, 665 (1990).

[3] Q.-F. Sun, J. Wang, H. Guo, Phys. Rev. B 71, 165310 (2005).

[4] P. Stefański, J. Phys., Condens. Matter 22, 505303 (2010).

[5] P.A. Orellana, M. Amado, F. Domínguez-Adame, Nanotechnology 19, 195401 (2008).

[6] F. Mireles, G. Kirczenow, Phys. Rev. B 64, 024426 (2001).

[7] Ł. Karwacki, P. Trocha, J. Barnaś, J. Phys: Condens. Matter. to be published.

[8] T.A. Costi, V. Zlatić, Phys. Rev. B 81, 235127 (2010).

[9] D. Boese, R. Fazio, Europhys. Lett. 56, 576 (2001).

[10] M. Krawiec, K.I. Wysokiński, Phys. Rev. B 73, 075307 (2006).

[11] G. Kotliar, A.E. Rückenstein, Phys. Rev. Lett. 57, 1362 (1986).

[12] B. Dong, X.L. Lei, J. Phys., Condens. Matter 14, 11747 (2002). 